\title{
Smart Textiles and Wearable Technologies for Sportswear: A Design Approach
}

\section{Venere Ferraro *}

Design Department, Politecnico di Milano, Via Durando 38/A, Milano, Italy

* Author to whom correspondence should be addressed; E-Mail: venere.ferraro@polimi.it Tel.: +39 022399 5933; Fax: +39-02-2399-5986.

Published: 12 November 2015

\begin{abstract}
The lately huge development of information technologies (ICT), wearable technologies and smart textiles has changing the way to conceive sport and well-being concepts. New kind of performing textiles, especially the electronic ones are nowadays used in several applications thought all everyday activities. The textile has become the supporting platform to integrate the technologies, add more functions to what we wear and exploit high-tech innovation. In this way, the textile, also adding computational technologies into, is "smart" and allows people to be interactive. We assist to a shift into textile from a static dimension to a dynamic one that consequently creates new dynamic products. This switch into the textile structure adds functionality but also create new behavior. The paper will describe the results of an educational activity carried out inside the Sportswear Studio Lab of Master Degree in Fashion Study at School of Design of Politecnico di Milano. The students were asked to generate a new advanced concept for sport application exploiting the potentiality of smart textile and wearable technology. The projects developed by the students followed a design process suggested by the author that requires the understanding of: (i) the what (the purpose of the concept); (ii) the how (the used technology); (iii) the where (the context in which the product is used) and finally (iv) the wearability issues connected to the role of technology in human body changing and perception.
\end{abstract}

Keywords: wearables; smart textile; wearability; design approach

\section{Introduction}


The term Wearable Technologies, 'fashionable electronics', or 'smart garments', is associated to those clothing and soft or hard accessories which integrate electronic components, or which are made of smart textiles. Forms and Technologies of wearables fall mainly into two categories: soft/hard supports; soft/hard electronics. Smart textiles are the results of a disciplinary approach that creates an intersection and overlapping of researches in different fields such as textile, design and technology, chemistry, physics, material science and computer science and technology.

The application of smart textiles sees its exploitation in the development of three research areas: conductive materials, miniaturized electronics (integration of electronics into textiles and products) wearable technologies with the use of wireless communication (to allow both people and device communication) [1].

A smart "wearable" textile system is a simple platform able to sense both human body and environment thanks to the presence of different kinds of sensors. A wearable, as the word itself expresses, is a device worn on the human body that incorporates the intelligence (sensors and electronics) into the clothes. This typology of device is able to sense, communicate, navigate but also actuate other devices. When we use the term smart textile, we refer to a textile platform that senses and reacts to human body or external environment without electronic control. Summarizing a wearable/smart device/textiles allow the user to wear contemporary both electronics and common clothes.

Such systems are made up of sensors/actuators and communicative systems thanks to which the user can assess to information about his personal biophysical data or and environmental ones.

The research field of wearable and smart textiles encloses different disciples such as biology, physiology, physics, chemistry, micro-nanotechnologies and material sciences, industrial sectors like medical devices, electronics, microchips, textile, telecommunications and engineering disciplines. [2]

Wearable technologies and smart textiles cover a very wide fields of applications: [i] Monitoring body vitals for health (stress levels, sleeping patterns, heart rate); [ii] Sport purposes (fitness level, performance optimization); [iii] Source of light (for entertainment purposes); [iv] Augmented reality (such as Google Glass, which project digital information into our field of vision).

With the development of interactive solutions integrated both into human body and wearable devices the boarders between human body and technology is getting subtle.

Nowadays "integration" of human and technology is an exploitation of what we have seen in the fiction/cyborg movies in the 60s. Today we assist to a self-regulating human-machine system (Manfred Clynes and Nathan Kline) "The human skin is an artificial boundary: the world wanders into it, and the self wanders out of it, traffic is two way and constant". [3]

The skin mentioned by Wolfe, is the technology itself, and is our intelligent covers, the platform that connect us with the surrender.

In this way the human body becomes the "media" for the interaction and consequently, the working machine equipped with technology, influenced by the technology but without losing its corporal essence. It, instead, reshapes its form in seek of perfection through the homologation (i.e prostheses)

Thanks to the technological progresses the human body increases its capability to sense. [4]

\section{Methodology}

\subsection{General Aim}


This paper aims at describing the results of an educational activity carried out inside the Sportswear Studio Lab of Master Degree in Fashion Study at School of Design of Politecnico di Milano. The workshop here described covers the theme of the wearable devices and smart textile. The focus of the workshop was on two main aspects: wearable technologies/smart textiles and tools to design them.

The objective of the workshop was for the students to: [i] be aware of available technologies and how to exploit them in fashion system; [ii] use wearability tools to enhance designers sensibility in shaping the technology in a desired/pleasured way for the users.

Students were asked to focus more attention to the achievement of wearability (wear-ability) that concerns the relationship between physical form of the wearables and the human body.

This is a very important aspect cause if well-investigated let the device get the essence of the paradigm: anytime, anyplace, by anyone. In this way a wearable system becomes an element able to follow movement in a totally and unobtrusive harmony with human body.

Besides, wearables shapes assumes an importance for the end-user culture and profile but also for the context ( i.e sport, work, leisure)

\subsection{Design Process}

The students were asked to generate a new advanced concept for sport application exploiting the potentiality of smart textile and wearable technology. The projects developed by the students followed a design process suggested by the author that requires the understanding of: (i) the what (the purpose of the concept); (ii) the how (the used technology); (iii) the where (the context in which the product is used) and finally (iv) the wearability issues connected to the role of technology in human body changing and perception.

Students were asked to be in-group and follow some steps in the development of their concept. In order to achieve the assignment they first had to chose a purpose among those provided by the teacher (the what): prevention, self-motivated, keeping fit for autonomy, keeping fit for thriving, competition, self-improvement, social and physical progress.

They then had to find a field of applications (the where) between: motorcycle, cycling, running, hockey, dancing, skiing but they could also proposes other interesting field of intervention. The chosen field had to match with the purpose and generate the goal of their brief. In the brief each group had also to identify what kind of technology to use in their concepts such as: smart materials, e-textile, embedded sensors, wearable technologies (the how).

Finally author suggested the students to use a tool that is a ri-elaboration of two studies developed in cross-disciplinary fields: the design guideline to develop wearable products ideated by The Institute for Complex Engineered Systems (ICES) and the langer lines used in surgery.

ICES found the most unobtrusive areas for wearable objects: (see fig. 1 left). Starting from these studies author identified a surgical theory, which provided a scientific approach to wearables. Author believes that the identification of design guidelines could be improved by matching the unobtrusive areas with Langer's Lines. A Langer line, called also cleavage lines, are lines along which there is less skin tension (see fig. 1 - right).

Hence during the concept generation students used the overlapping of the Langer Lines and ICES' non-obtrusive areas to develop their ideas. 


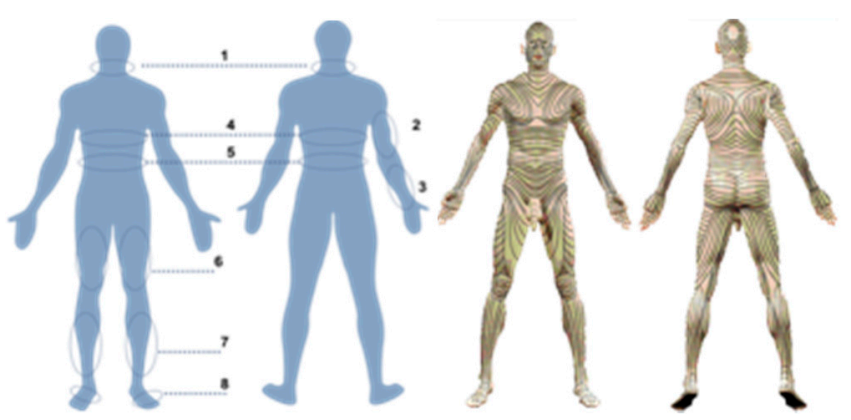

Figure 1. Ri-elaboration of unobtrusivity areas by Gemperle at al. (left) Langer's Lines (right).

\section{Results and Discussion}

Hereafter three projects selected by those developed by the students are presented: 1) A glowing sweatshirt for little ice skaters; 2) A tracking safety kit; 3) A Lightning gloves for spinners.

The glowing sweatshirt was generated with the purpose to motivate children in practicing iceskating. The idea was to design a shirt, the most useful garments for ice skating training, and the most versatile, equipped with the LEDs and GPS that measure the activity done by the child. The lines of LEDs glows up gradually thanks to an electronic controller connected to a GPS and a LED system.

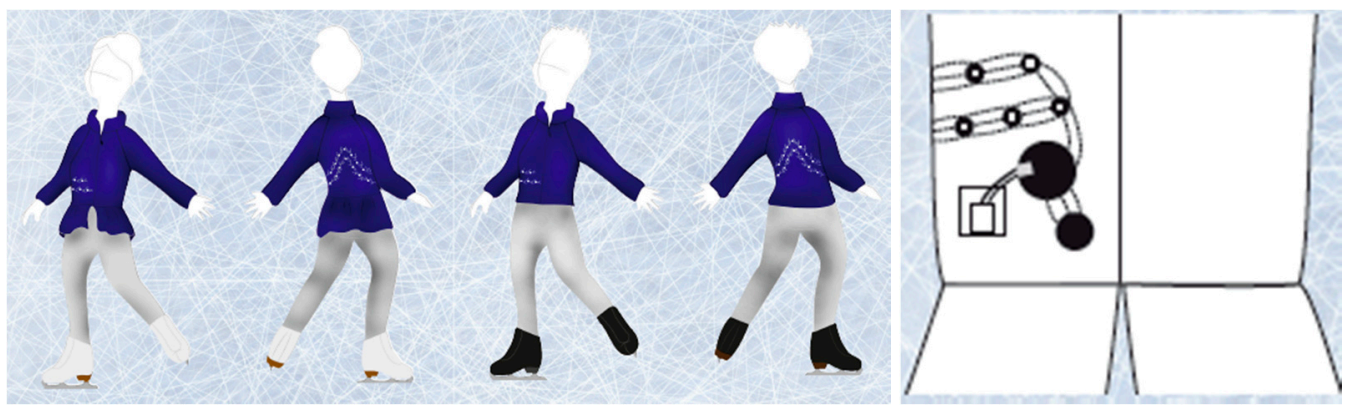

Figure 2. The Glowing Sweatshirt.

The students that developed the tracking safety kit set the objective of keeping the users safe in the darkness and in dangerous situation connecting each others

The kit is made up of two products: a pair of shoes (able to lights up the path during the night) and a bracelet (to communicate).

The shoes are equipped with a strip of LEDs connected to a rechargeable battery that consists of piezoelectric component, capacitor and rectifier connected to a pressure sensor and GPS in order to convert kinetic energy in electric energy.
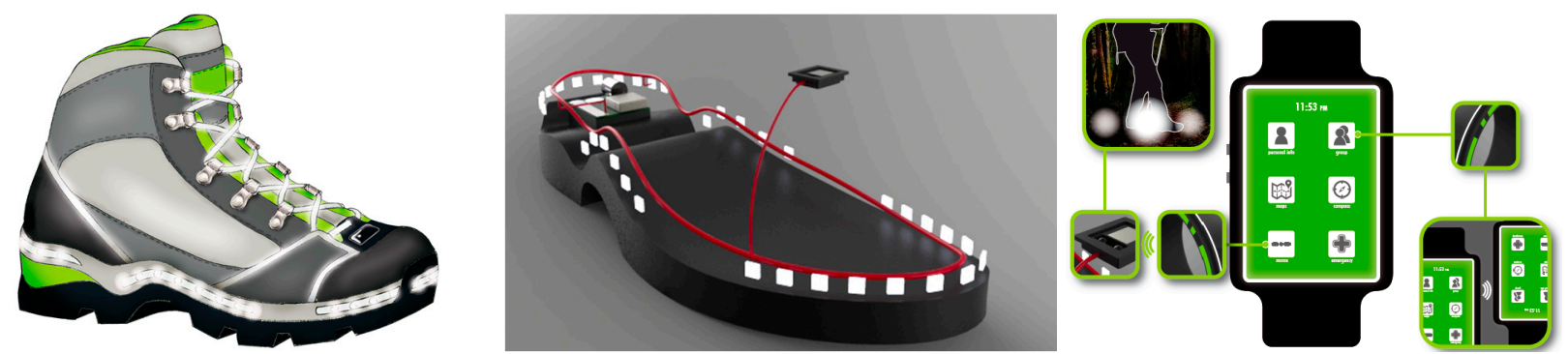

Figure 3. A tracking safety kit. 
The lightning glove is made up of a heart rate monitor that detects the heart rate from the wrist. It was developed with the aim to self-motivate the users during the activity of spinning. The heart rate monitor is connected to a screen where users can set different parameters according t 3 different intensity workouts. It is also connected with five LEDs set on the glove. LEDs light up in green when the heart rate remains inside the parameter set, while LEDs light up in red when the parameters fall or rise above the threshold.

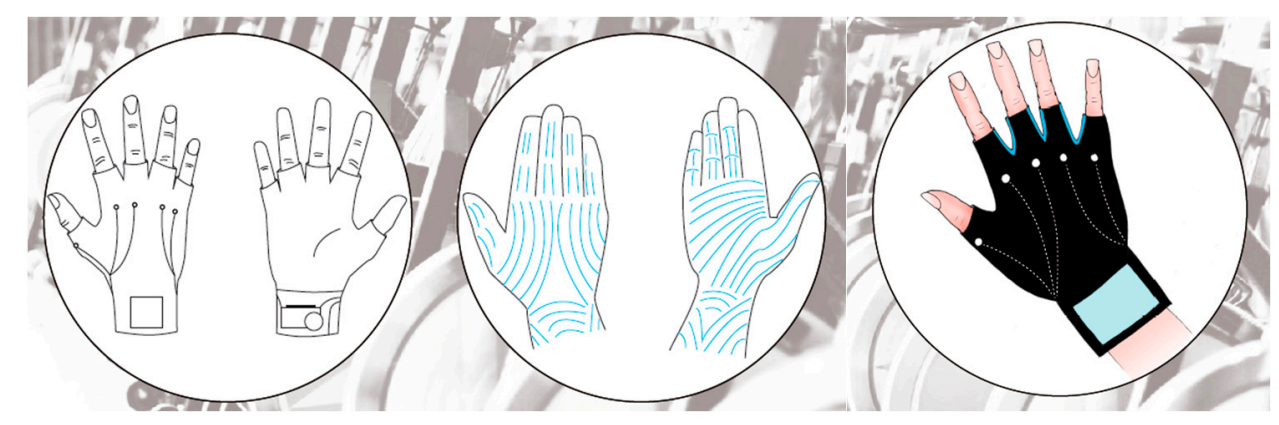

Figure 4. A tracking safety kit.

These projects were selected according to the level of understanding of the topic (wearable technologies and smart textile) the level of awareness of available technologies and how to exploit them in fashion system in order to obtain a good trade off between the hard (technology) and the soft (the fabric).

\section{Conclusions}

The experience of the workshop before mentioned, gave the author the opportunity to reflect on teaching choices and tools. Dealing with technology was a new experience for the students attending a master degree in Fashion System. Despite that, the subject of the workshop, which at first might seem very challenging for students, was truly engaging for them. Their participation to the course was active and enthusiast through the entire workshop. This asset is proved by the positive final feedback we receive form the course. Nevertheless author realized that a multidisciplinary approach that covers also engineering expertise is needed in order for the designer to exploit and applied new technologies for the development of smart wearable systems able to extend human limits and create new experiences.

Besides, an overview of the user (gender, age group) for whom the product is designed for it is needed. Indeed a deep analysis of the user let the designer understand the human physiological issues but also the functional ones in order to address comfort in everyday life.

\section{Acknowledgments}

Author wants to thank students from the Master Degree in Fashion System that developed the projects presented in this paper.

\section{Conflicts of Interest}

The author declares no conflict of interest. 


\section{References}

1. Berglin, Lena. 2013. Smart Textiles and Wearable Technology - A study of smart textiles in fashion and clothing. A report within the Baltic Fashion Project, published by the Swedish School of Textiles, University of Borås.

2. Lymberis A. (2003) Intelligent biomedical clothing for Personal Health and Disease Management: State of the Art and Future Vision, Telemedicine journal and e-health volume 9, number 4, 2003

3. Wolfe, B.. Limbo. Carroll \& Graf Publishers, 1987

4. Fiorani E., Abitare il corpo: la moda, Lupetti, 2003

5. McCann J., Bryson D. (edited by) Smart Clothes and wearable technology, Woodhead Publishing in Textiles, feb 2009

6. Dunne L., McCann J., Mörsky S. (2006), Human a Tutorial, Tenth International Symposium on Wearable Computers (ISWC 2006), Montreux, Switzerland, October 11-14.

7. Seymour S. (2008), Fashionable Technology. The Intersection of Design, Fashion, Science and Technology, Springer- Wien, New York.

(C) 2015 by the authors; licensee MDPI, Basel, Switzerland. This article is an open access article distributed under the terms and conditions of the Creative Commons Attribution license (http://creativecommons.org/licenses/by/4.0/). 